\title{
Micromechanics of complex granular materials: a focus on small strain be- havior
}

\author{
V. Magnanimo* \\ ${ }^{1}$ MultiScale Mechanics, ET, MESA+, University of Twente, Enschede, Netherlands
}

\begin{abstract}
We study the bulk properties of isotropic and anisotropic granular assemblies using discrete element simulations and experiments. The focus is on the influence of the microstructure on the elastic properties of the aggregate. By studying three selected examples, namely assemblies of monodisperse glass beads, bidisperse mixtures in size and soft-stiff bimodal mixtures, we show that the effective moduli of a dense granular assembly can be manipulated by properly combining material characteristics and system properties.
\end{abstract}

\section{Introduction}

Granular materials behave differently from usual solids or fluids and show peculiar mechanical properties like dilatancy, history dependence or ratcheting due to their intrinsically discrete nature. To understand the behavior of granular materials it is first necessary to characterize their state by introducing an appropriate set of (independent) internal variables. In early studies, macroscopic variables measurable in laboratory experiments, i.e., stress and porosity, were thought to be sufficient to characterize the elastic stiffness of a granular assembly, see [4] and references therein. However, with further investigations it has been observed that other factors are also important $[1,8,10,14,16]$. Among those are the geometric arrangement of the grains, i.e., the geometric fabric, and the interparticle forces, probability distributions and correlations and in particular the concept of weak and strong force network. There are different mechanisms that lead to a change of the structure. When a granular assembly is deformed, neighboring particles may form new contacts and, at the same time, some existing contacts may disappear, i.e., a geometric fabric develops [15]. On the other hand, collective deformations of many grains can lead to re-arrangements on a larger scale, (non-affine), which also brings change to structure. While the former local contact creation or destruction is closely related to the compressive and tensile directions of strain, the latter phenomenon is at the origin of the interesting behavior of granular media.

In many industrial applications as well as in natural phenomena, granular assemblies are constitute not only by one type of particles but by of combination of them. Processes involving milling, segregation, agglomeration, filtration and sieving are common and often lead to the generation of granular systems with large size ratios. Dealing

\footnotetext{
^e-mail: v.magnanimo@utwente.nl
}

with mixtures systems is exceptionally challenging and often requires heuristic assumptions to be made, as prediction/control of the behavior is still an unsolved issue. A common and interesting sub-class are bimodal granular mixtures. When two types of particles are mixed (homogeneously), the granular material displays a peculiar macroscopic behavior due to the non-trivial arrangement of the two species. The behavior is non-linear and often non-monotonic, in some cases showing dramatic sudden changes. For example, it has been well established that deformation of powder compacts can be severely retarded by the presence of heterogeneities such as inclusions or reinforcements, where the role of the volume fraction and shape of reinforcements on the densification behavior needs to be further highlighted [9]. A well known example is the binary composite consisting of a conducting phase and an insulating phase used for electromagnetic shielding and anti-static devices. These composites show a sharp transition from low to high conductivity at a critical concentration of the conducting phase [2]. It is well known in the geomechanical community that the presence of fines strongly influences the mechanical behavior of granular soils. Scientific work on the topic is extensive and several studies have de-scribed the dependence of elastic bulk stiffness and stress-strain behavior of granular mixtures on the volume of fines (see [20] and references therein).

Despite the massive amount of work on granular mixtures, a deep understanding from a micromechanical perspective is still missing. which experimentally is difficult to achieve for very small particles. This is because the role of the different components can not be discerned by classical experiments. Here we combine discrete element simulations and experiments to highlight the influence of the microstructure on the effective elastic stiffness of monondisperse and bidisperse granular assemblies under isotropic and anisotropic conditions. We present a set of examples, namely monodisperse granular assemblies 
under difference stress paths, bidisperse mixtures with different size and bimodal mixtures made of soft-stiff particles We show that the bulk properties of the material can be controlled by indirectly tuning the microstructure, where increasing complexity offers more degree of freedom.

\section{Single specie samples}

In order to investigate the relation between elasticity and microstructure, we use the distinct element method [3] and prepare random assemblies of identical, frictional, elastic spheres. We deal with aggregates of 10,000 particles with radius $R=0.1 \mathrm{~mm}$ randomly generated in a periodic $\mathrm{cu}$ bic cell. We employ material properties typical for glass spheres: a shear modulus $G_{g}=29 \mathrm{GPa}$ and Poisson's ratio, $v=0.2$. The interaction between particles is a noncentral contact force in which the normal component follows the non-linear Hertz's law. For the tangential component we incorporate a bilinear relationship with elastic displacement followed by Coulomb sliding with friction coefficient $\mu$ (details are given in [12]).

\subsection{Isotropic samples}

After random generation, particles are isotropically compressed in the absence of gravity from an initial gas to the desired solid volume fraction. Because we are interested in dense aggregates of frictional particles, we require that the solid volume fraction $\phi$ is close to the random close packing value $\phi_{R C P} \simeq 0.64$. It is well known that different packing structures are obtained experimentally according to the preparation protocol. Our approach is to generate numerical packings with different final structure. To do this we employ a protocol where $\phi$ and $p$ are set in two different stages of the deformation (details are given in [14]). The result is that, for a given pressure $(p=100 \mathrm{kPa})$ and identical solid volume fraction $(\phi \simeq 0.638)$, we obtain isotropic packings with different coordination number, that is the average number of contacts per particle $Z_{0}^{*}$ [19]. Here we consider three aggregates with initial coordination number $Z_{0}^{*}=6.17,5.36,4.88$ respectively.

\subsection{Axialsymmetric compression}

The three samples described above are subjected to axisymmetric deformation at constant mean stress $p=p_{0}=$ $100 \mathrm{kPa}$ [19]. During each loading step, the target pressure is maintained with a servo mechanism which continuously adjusts the applied strain rate according to the difference between the desired stress state and the stress measured at that time step [14]. In order to reproduce a quasi-static, triaxial loading we compress the sample very slowly $\left(\gamma_{\partial t} / \Delta_{0}\right.$ is lower $5 \times 10^{-3}$, where $\gamma_{\partial t}$ is the accumulated strain in the time step $\partial t$ ). After each increment we let the system to relax until a new equilibrium state is reached.

\subsection{Elastic moduli}

For each one of the isotropic samples in Sec.2.1 as well as $\mathrm{tt}$ different steps along the axisymmetic loading paths

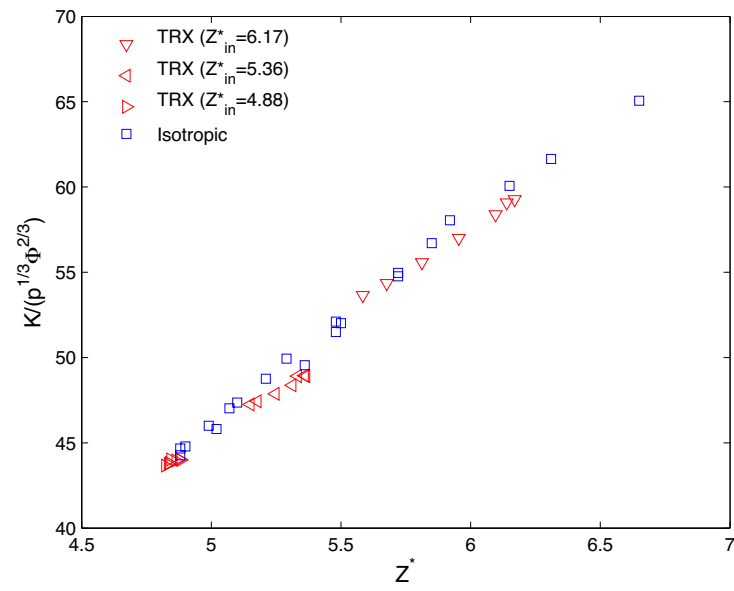

Figure 1. Evolution of the normalized bulk modulus versus coordination number, for axisymmetric compression (TRX) of the three samples with different initial microstructure $Z_{0}^{*}$ and for isotropic samples.

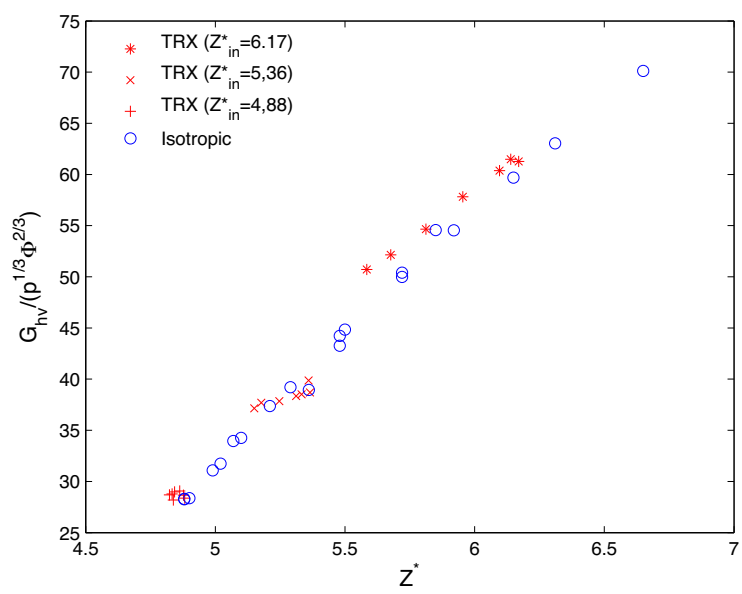

Figure 2. Evolution of the normalized shear modulus versus coordination number, for axisymmetric compression (TRX) of the three samples with different initial microstructure $Z_{0}^{*}$ and for isotropic samples.

in Sec.2.2, we estimate the elastic stiffness of the granular assemblies. We focus on two moduli, the bulk modulus $K$ and the axial shear modulus $G_{h v}$. They are calculated by applying an incremental strain and measuring the corresponding incremental stress response [13, 14]. We apply a shear strain, $\Delta \epsilon_{h v}$ and measure the stress response $\sigma_{h v}$ to obtain $G_{h v}$. We also apply an isotropic strain, $\Delta v=\Delta \epsilon_{11}+\Delta \epsilon_{22}+\Delta \epsilon_{33}$, to measure the bulk modulus $K$, where $\epsilon_{i j}$ are components of the strain tensor. The friction coefficient is set on a very high value to prevent sliding between grains because we are interested in the elastic resistance of the aggregate. For each calculation, we verify that the applied strain is small enough to be in the linear response regime. 
In Fig. 1 and Fig. 2 the bulk and shear moduli are collected for all numerical packing and plotted against the coordination number. The moduli are normalized with a power of pressure and volume fraction, $p^{1 / 3}$ and $\phi^{2 / 3}$, following the prediction of effective medium theory [21]. In this ratio, the dependence on pressure and volume fraction cancel out and all that remains is the relation with the actual value of coordination number $Z^{*}$. Surprisingly, the plots of $B\left(Z^{*}\right)$ and $G_{h v}\left(Z^{*}\right)$ result in universal curves, irrespective of induced anisotropy or (deviatoric) stress state, as predicted by classical theories [17]. That is, once the actual state has been achieved, the measurement of the bulk and shear stiffness can be associated with a unique coordination number, which acts as a state variable. This is able to characterize the microstrusture of any granular sample, along with pressure and void ratio, no additional specification of the stress states needed. It is noteworthy that the major role is taken by the scalar coordination number for both isotropic and anisotropic cases, while the orientation of contacts (fabric tensor) most likely plays second-order effects.

\section{Toward complexity}

\subsection{Bidisperse mixtures of particles with different sizes}

As further step toward complexity, we look at how the elastic properties properties of a monodisperse, isotropic granular assembly vary in the case of a bidisperse mixture, with the main focus on the bulk modulus. In order to keep the discussion as simple as possible, we limit ourself to the case where only $5 \%$ of the volume is substituted with particles of different size (and same characteristics). We analyze the properties of the granular mixture on two phase spaces: (i) by varying the size ratio of fines to coarse and (ii) by spanning a wide range of volume fraction, and find the optimum size ratio that maximizes the bulk modulus at each volume fraction.

We use again a numeric approach via DEM to discuss the mixture behavior. The reference sample consists of $N_{A}^{0}$ monodisperse particles $A$ of radius $r_{A}$. Starting from this base sample, many mixtures are created by substituting a given number of of $N_{A}^{0}-N_{A}^{T}$, with particles of species $B$ of different radius $r_{B} \leqslant r_{A}$, such that the same volume $\left(N_{A}^{0}-N_{A}^{T}\right)(4 \pi / 3) r^{3} A=N_{B}^{T}(4 \pi / 3) r^{3} B=V_{B}^{T}$ of material $A$ is replaced by $B$. Further details are given in [7]. For the sake of simplicity a linear contact model for frictionless particles is used in this part of the study. However details of the implementation do not affect the results.

After preparing isotropic samples at different values of volume fraction, $\phi=0.69-0.82$ per each size ratio $r_{B} / r_{A}$, the bulk modulus is calculated in a similar fashion as in Sec.2.3. Fig.3 shows the evolution of the bulk modulus scaled by $2 r_{A} / k$, with $k$ contact stiffness, plotted against size ratio for different volume fractions. As expected, $K$ increases systematically with density. For loose states ( $p h i=0.69,0.72), K$ mostly increased with increasing $r_{B} / r_{A}$. The behavior associated with denser states is much more interesting, as we observe an increase in $K$

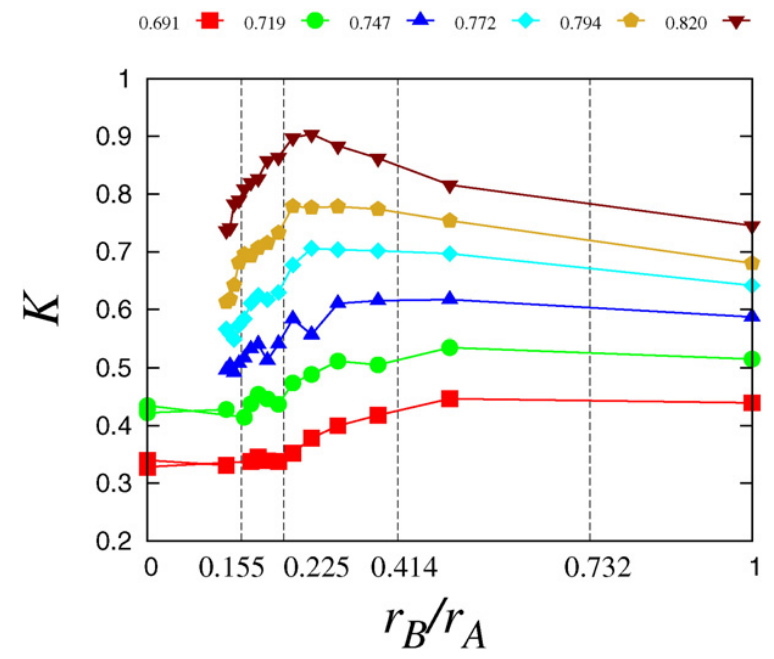

Figure 3. Evolution of the bulk modulus normalized by $2 r_{A} / k$ versus size ratio for bidisperse mixtures at different values of volume fraction (from [7]).

to a maximum, followed by a decrease for larger $r_{B} / r_{A}$. Note that the value of $r_{B} / r_{A}$ where $K$ reaches its maximum varies with increasing densities and the maximum becomes stronger. From Fig.3, we extract very important insights: (i) The bulk modulus of a granular assembly can be manipulated by only substituting $5 / 105 \%$ of the base material with fines. (ii) We can control the direction of the change (enhanced or lowered bulk modulus) and the magnitude of change through the density and the size of the small particles. (iii) For dense material, for a given density, there is an ideal size of fines that leads to the maximum in the bulk modulus.

Interestingly, we see in [7] that the trend observed for $K$ in Fig. 3 is mainly related to the behavior of $A B$ interactions between small and big particles, while the actual value depends on the contributions of $A A$ main network. We associate the different trends observed for loose and dense systems with the ability of the fines (material $B$ ) to fill the voids formed by particles A. With increasing density, the void size gets smaller and compatible with the size of particles $B$, and thus contribute to the active contact network. For dense assemblies, $K$ attains maxima near the size ratio corresponding to tetrahedron configurations, meaning that the dense state is more likely (possibly due to ongoing crystallization) to create such a configuration and this is the efficient mean arrangement. For the looser systems, the maximum of $K$ moves towards higher size ratios $r_{B} / r_{A}$, corresponding to cubic-like configurations.

Hence the bulk stiffness of a granular material is strongly affected by the addition of a different component (even in a very small amount) that leads to modifications in the microstructure of the main component. The interest arises from geophysical hazards, like earthquakes, where the material volume remains constant, but the size of few particles can decrease quickly due to breakage. The change in the size distribution, even limited to a very small volume, has been shown to play an important role 
in soil stability. Important applications exist for example in pharmaceutical and food processes such as chromatographic separation or extraction, where the flow through a deformable granular material depends strongly on the effective bulk modulus and can be enhanced by by adding inclusions to the main particle consistent. In these industrial processes the focus is optimizing the performance of a given (granular) material with minimum modification, minimum costs. Other obvious applications of this study cover building mixtures like concrete or asphalt, and shock absorbing materials. The presence of small particles in a granular mass, which is often associated with weakness, can be an asset for functionality.

\subsection{Bimodal mixtures of soft-stiff particles}

Finally we want to highlight the influence of particle materials on the elastic behavior of a granular mixtures. To address this topic, we have performed wave-propagation experiments on isotropic mixtures particles of two different species, namely glass and rubber beads, but identical size. Details of the experiments are reported in [18]. Results are consistent with evidences by previous studies $[6,11]$ and show how granular mixtures can be engineered to exhibit exceptional properties.

When we look at the evolution of the elastic modulus with rubber content and pressure, we observe that, similarly to bidisperse mixtures in Sec.3.1, the bulk behavior can be tuned by a proper combination of material parameters and state variables, that eventually lead to modifications of the glass network microstructure.

The elastic stiffness decreases non-linearly with rubber content $v$. The modulus is fairly constant for $v \lesssim 30 \%$, decreases sharply between $30 \% \lesssim v \lesssim 70 \%$ and is again independent of rubber content for $v \gtrsim 70 \%$. In the case of high pressure, adding a small amount of soft particles surprisingly enhances the effective stiffness of the medium and the highest modulus is observed at $v \simeq 20 \%$, indicating that a mixture of $20 \%$ rubber content may become stiffer than a pure glass aggregate. That is proper mixtures of soft and stiff particles exhibit enhanced stiffness along with low porosity, high compressibility and high attenuation. Eventually, the combination of these properties is highly desirable in engineering practice for many constructions applications, including highway embankments or backfill for retaining walls [6].

\section{Conclusion}

We have used DEM simulations and experiments to analyze the bulk properties of granular assemblies, in the cases of i) monodisperse particles under isotropic/anisotropic stress paths, ii) isotropic mixtures bidisperse in size and iii) soft-stiff isotropic mixtures. We have focused on the effective elastic moduli, measured by applying small perturbations to the system. We have shown that the microstructure controls the bulk stiffness and can be manipulated to enhance the material properties as useful to contrast natural hazards or for industrial applications. In particular, the scalar coordination number is the main parameter that characterize bulk and shear moduli for isotropic and anisotropic aggregates of monodisperse particles. In the case of bidisperse mixtures, while $K$ decreases monotonically for loose systems, for dense systems, $K$ first increases, reaches an optimum, and later decreases, the peak value depending on the density of the mixture, that is the optimal geometrical arrangement of the particles. Finally glass-rubber mixtures show an enhanced bulk stiffness in combination with low density and high damping for small amount of rubber. An interesting extension of this study would consider a hierarchical granular medium in which the particle size distribution, type and eventually shape [5] are engineered to obtain desired mechanical properties, e.g. the bulk or shear modulus.

\section{References}

[1] I. Agnolin and J. N. Roux, Phys. Rev. E 76, 061304 (2007).

[2] C.-H. Kuo and P. K. Gupta Acta metall. mater. 43(1), 397403 (1995).

[3] P. A. Cundall and O. D. L. Strack, Géotechnique 29, 47 (1979).

[4] J. D. Goddard, Proc. R. Soc. Lond., Ser. A 430, 105 (1990).

[5] M.Z. Miskin and H.M. Jaeger, Nature Materials 12, 326 (2013).

[6] H.-K. Kim and J. C. Santamarina, Can. Geotech. J. 45(10), 1457 (2008).

[7] N.Kumar, V. Magnanimo, M. ramaioli and S. Luding, Powder Tech. 293, 94 (2016).

[8] R. Kuwano and R. J. Jardine, Géotechnique, 52, 727 (2002).

[9] D. Bouvard and F. F. Lange Acta metall. mater. 39(12), 3083 (1991).

[10] L. La Ragione and V. Magnanimo, Phys. Rev. E 85, 031304 (2012).

[11] C. Lee, Q. H. Truong, W. Lee and J.-S. Lee, J. Mat. Civ. Eng. 22(4), 323 (2010).

[12] H. A. Makse, N. Gland, D. L. Johnson, and L. Schwartz, Phys. Rev. Lett. 83, 5070 (1999).

[13] H. A. Makse, N. Gland D. L. Johnson, and L. M. Schwartz, Phys. Rev. E. 70, 061302 (2004).

[14] V. Magnanimo, L. La Ragione, J. T. Jenkins, P. Wang, and H. A. Makse, Europhys. Lett. 81(3), 34006 (2008).

[15] M. Oda, Soils Found. 12, 17 (1972).

[16] F. Radjai, D.E. Wolf, M. Jean, J. Moreau, Phys. Rev. Lett. 8061 (1998).

[17] S. Roesler, J. Geotech. Eng. Div. 105, 871 (1979).

[18] K. Taghizadeh, H. Steeb, V. Magnanimo, and S. Luding, In this volume (2017).

[19] C. Thornton, and S. J. Antony, Roy. Soc. of London Phil. Tr. A 356, 2763 (1998).

[20] L.E.Vallejo, Can. Geotech. J. 38, 1097 (2001).

[21] K. Walton, J. Mech. Phys. Solids, 35, 213 (1987). 